\title{
Impacto personal y escolar del embarazo y maternidad en estudiantes universitarios
}

Gloria Ángela Domínguez Aguirre, ${ }^{1}$ Adriana Martínez Peralta, ${ }^{1}$ Héctor Herrera Barragán ${ }^{1}$ y María Elena Rincón Aguirre ${ }^{1}$

\section{Introducción}

El embarazo y maternidad son aspectos considerados como deseables una vez que la mujer ha concluido con sus estudios universitarios, ha obtenido una independencia económica, ha disfrutado de una variedad de actividades de ocio y tiene una estabilidad en la relación con su pareja; sin embargo, de acuerdo con el Instituto Nacional de la Mujer (2013), 87.7\% de los nacimientos ocurridos en el año 2012 corresponden a madres con escolaridad máxima de preparatoria; y $11.5 \%$, a mujeres con estudios de licenciatura.

Por otra parte, las estadísticas del Instituto Nacional de Estadística y Geografía (INEGI, 2013) refieren que $46.6 \%$ de los nacimientos registrados en el año 2012 corresponden a mujeres entre 15 y 24 años, periodo de edad de grandes transformaciones en el que se deberían realizar los estudios de nivel medio superior y superior, determinantes en el tránsito hacia la vida productiva.

1 Universidad Juárez Autónoma de Tabasco. 
En las mujeres universitarias, la vivencia de un embarazo, con la consecuente maternidad, impacta profundamente en su vida en lo que se refiere a las relaciones con su familia, pareja y amigos, así como en su vínculo con los escenarios en los que se desenvuelve, principalmente, la escuela.

Al respecto, Hernández y Orozco (2011) encontraron que la mayoría de las estudiantes universitarias que fueron madres se encontraban solteras después del embarazo, tenían el apoyo por parte de la familia y el personal docente de la universidad donde estudiaban; a la mitad no las apoyaba la pareja en el cuidado de su hijo y no se sentían preparadas para ser madres.

Estupiñán y Rodríguez (2009) estudiaron los aspectos psicosociales relacionados con el embarazo en 68 estudiantes universitarias que tuvieron un embarazo adolescente, encontrando que se generan crisis y ajustes en la estructura familiar y afectiva. La madre de la gestante es quien apoya permanentemente a su hija; el acompañamiento de la pareja se da durante los primeros meses, seguido por abandono.

La madre de la estudiante se convierte en el apoyo principal y es quien comunica al padre el embarazo y quien se encarga del cuidado del bebé durante la jornada escolar de la estudiante. El apoyo brindado por la escuela se considera escaso, lo cual se agrava cuando no se tiene el apoyo de la familia y la pareja (García, Acuña y Preciado, 2005).

\section{Método}

\section{Participantes}

Participaron 58 estudiantes del área de Ciencias de la Salud de la Universidad Juárez Autónoma de Tabasco. Como criterios de inclusión se consideraron las estudiantes que han vivido un embarazo con un nacimiento vivo y que actualmente estén desempeñando su rol de madre o lo hayan hecho en el transcurso de sus estudios universitarios. 


\section{Material}

Encuesta compuesta de 50 ítems, con cuatro opciones de respuesta (casi nunca, algunas veces, muchas veces, casi siempre), que mide el apoyo recibido por la familia, el apoyo recibido por la pareja, el apoyo recibido por la escuela (docentes, compañeros y tutor), así como el impacto del embarazo y la maternidad en su estado de ánimo, vida personal y rendimiento escolar. Esta encuesta fue procesada con SPSS V.15.

\section{Procedimiento}

Se realizó un muestreo por conveniencia para efectuar un estudio de tipo descriptivo y exploratorio orientado a identificar el impacto de la vivencia del embarazo y maternidad en la vida personal y escolar de las estudiantes de una universidad pública, así como el apoyo familiar, escolar y de la pareja.

\section{Resultados}

De las 58 estudiantes universitarias participantes, $73.2 \%$ están casadas o viven en unión libre; $17.9 \%$ son solteras y $8.9 \%$ están separadas o divorciadas; contrario a lo encontrado por Hernández y Orozco (2011), pues según ellos la mayoría de las estudiantes estaban solteras; $67.2 \%$ provienen de familias con padres casados; $24.2 \%$, de familias con padres divorciados o separados; $5.2 \%$, de una familia monoparental (madre); y 1.7\%, de una familia adoptiva; $70 \%$ menciona que el padre de su hijo(a) y ella son pareja, aunque sólo $60.3 \%$ viven juntos; $25.9 \%$ vive con sus padres, $8.6 \%$ con sus suegros, $3.4 \%$ con un familiar y $1.7 \%$ vive sola.

$8.6 \%$ de las estudiantes encuestadas tenían entre 15 y 18 años en su primer embarazo; 74\%, entre 19 y 22 años; y 17.4\%, más de 23 años. En cuanto a la edad de la pareja, a diferencia de las mujeres, ésta se concentra en el grupo de edad de más de 23 años (53.5\%), seguido del grupo de edad de 19 a 22 años (36.2\%) y de 17 y 18 (10.3\%). 
De acuerdo al grado que cursaba cuando ocurrió el primer embarazo, $24.1 \%$ cursaba cuarto año; seguido de $20.6 \%$, que estudiaba segundo; 19\%, tercero; 13\%, primero; y 10.3\%, quinto año.

De las estudiantes, 74\% tiene un hijo; 19\%, dos hijos; y $6.8 \%$, tres hijos. El total de hijos de las estudiantes es de 77 , la mayoría (76.6\%) tiene menos de cinco años.

\section{Apoyo recibido por la familia}

La mayoría de las estudiantes (91.4\%) percibe que su familia le brindó un mayor apoyo durante la maternidad que durante el embarazo (68.9\%), recibiendo un alto apoyo de ambos padres en lo económico (74.5\%) y en el cuidado de sus hijos/as (62.2\%) para que puedan estudiar, por lo que desde la perspectiva de las jóvenes encuestadas, el apoyo de la familia nuclear es determinante para la conclusión de la carrera. Llama la atención que sólo $48.5 \%$ menciona haber recibido apoyo de la familia política durante su embarazo y maternidad. Se entendería que dadas las circunstancias generales de este tipo de embarazos, las redes de apoyo familiar dominantes proceden de la familia materna.

A 82.8\% sus padres las dejaron elegir lo que deseaban hacer respecto al embarazo y las apoyaron en las decisiones que tomaron. Sólo a $17.5 \%$ les recriminaron frecuentemente haber quedado embarazada a temprana edad o sin estar casadas.

Los padres (91.3\%) y familiares cercanos (79.3\%) se convierten en las personas que más motivan a las estudiantes para continuar sus estudios; sólo a $22.4 \%$ sus familiares les sugirieron abandonar la escuela para dedicarse al cuidado de los hijos.

Apoyo recibido por los compañeros de clases

En el caso de los amigos y compañeros, $81.1 \%$ menciona que recibió un alto apoyo y solidaridad de sus compañeros/as; para $74.1 \%$, el apoyo se vio traducido en la notificación de las tareas y trabajos para entregar; y para 13.8\%, en el cuidado de su hijo mientras ellas estaban en clases. 
Apoyo recibido por los docentes

Por otra parte, el apoyo que pueda brindar el docente también es importante en esta etapa de la vida de la estudiante; sin embargo, sólo $51.8 \%$ dice que el docente le daba la oportunidad de entregar tareas y/o presentar exámenes extemporáneamente, y en el caso del docente-tutor, sólo $24.1 \%$ menciona haber recibido su apoyo.

Apoyo recibido por la pareja

De las alumnas, $62 \%$ menciona que su pareja frecuentemente se mostró feliz ante la idea de ser padre, aunque sólo $50 \%$ acudió frecuentemente a las consultas médicas; $73.4 \%$ menciona que recibió el apoyo que esperaba por parte del papá de su hija/o, le brindó apoyo económico (72\%), acudió a reuniones o actividades del hijo/a (58.7\%), y cuidó del hijo/a para que ella pudiera estudiar (53.5\%).

De las estudiantes, $29.3 \%$ menciona que actualmente enfrenta la maternidad sin el apoyo del padre de su hijo/a.

Impacto del embarazo y la maternidad

en su estado de ánimo, vida personal y rendimiento escolar

En relación con el estado de ánimo de las estudiantes universitarias durante el embarazo y la maternidad, encontramos que $69.3 \%$ de las estudiantes consideran que su estado de ánimo no varió o cambió durante el embarazo; las principales emociones y sentimientos que generó el embarazo fueron: intranquilidad (43.1\%), desánimo (31\%), infelicidad (29.3\%), depresión (27.6\%), enojo frecuente (22.4\%), miedo (22.4\%), culpa $(20.7 \%)$ y sentimientos de soledad y abandono (8.6\%); $70.7 \%$ sintió que sería difícil cuidar a su hijo, aunque sólo 31\% sintió temor de no saber cómo educarlo. Durante la maternidad, 50\% de las estudiantes se sienten presionadas y encuentran dificultad para combinar el rol madre-estudiante.

La vivencia del embarazo y la maternidad impacta la forma de entender y vivir la vida; $91.4 \%$ considera que les ha hecho 
sentir que son capaces de afrontar grandes retos, se sienten más maduras (91.9\%), más responsables (89.6\%), les trajo muchas satisfacciones (87.9\%) y les otorgó un sentido de vida (82.7\%). Sólo $17.2 \%$ piensa que le hubiera gustado ser madre en otro momento de su vida.

Por otra parte, un porcentaje de las estudiantes considera que su rendimiento académico se vio afectado durante su embarazo: por faltar frecuentemente a clases $(15.5 \%$ de las alumnas), por dificultades para la realización de tareas escolares (27.6\%), por la caída de su estado de ánimo (22.4\%). En términos generales, $55.6 \%$ de las alumnas indica que les ha sido difícil atender sus responsabilidades académicas por desempeñar el rol de madre.

\section{Discusión}

Ser estudiante universitaria, vivir un embarazo y ser madre demanda habilidades y fortalezas personales, así como una serie de condiciones del entorno que permitan continuar con la meta académica establecida, ya que el riesgo de abandonar los estudios universitarios es muy alto. Ante este riesgo y dada la cantidad de alumnas que combinan su rol de madre y estudiante universitaria, la universidad no puede permanecer indiferente, se requiere establecer diversos apoyos dirigidos a estudiantes embarazadas y madres para que, dependiendo de su situación particular, puedan concluir sus estudios universitarios y así acceder a una mejor calidad de vida para ellas y para sus hijos.

Siendo que la representación directa de la institución universitaria (maestros y tutores) no tiene un impacto facilitador equiparable al de los demás actores relacionados con las estudiantes que viven este tipo de procesos, se sugiere la intervención de la universidad en la creación de grupos de autoayuda, implementación de programas de sensibilización frente al embarazo y la maternidad y organización de cursos especiales para la nivelación académica. 


\section{Referencias}

Estupiñán-Aponte, M. y Rodríguez-Barreto, L. (2009). Aspectos psicosociales en universitarias embarazadas. Revista de Salud Pública, 11(6), 988-998. Recuperado de http://www. redalyc.org.

García, M., Acuña, M. y Preciado, F. (2005) Repercusiones del embarazo y la maternidad en la vida académica de las estudiantes universitarias. Revista Género, 13(36), 125-132. Recuperado de http://bvirtual.ucol.mx.

Hernández, K. y Orozco, E. (2011). Embarazo en estudiantes de carreras universitarias. Dictamen Libre, 8(8), 68-72. Recuperado de http://www.unilibrebaq.edu.co.

Instituto Nacional de Estadística y Geografía (2013). Estadísticas a propósito del Día Mundial de la Juventud. Recuperado de http://www.inegi.org.mx.

Instituto Nacional de la Mujer (2013). Mujeres y Hombres en México 2013. 10-17 Recuperado de http://estadistica.inmujeres.gob.mx. 
